\title{
Research on Bi-level Coordinated Optimal Dispatching Strategy for Microgrid Cluster
}

\author{
He langchao ${ }^{1}$, Wu Lizhen ${ }^{1 *}$, Chen Wei ${ }^{1}$, Hao Xiaohong ${ }^{1}$ \\ ${ }^{1}$ College of Electrical and Information Engineering, Lanzhou University of Technology, Lanzhou, Gansu, 730050, China
}

\begin{abstract}
The interconnection of multiple microgrids can form a microgrid cluster (MGC). The economic benefit and operation reliability of the whole system can be improved through the energy mutual-aid and coordination control between microgrids. This paper proposes a bi-level coordinated optimal dispatching strategy for microgrid clusters. The upper layer aims to minimize the operating costs of the microgrid cluster system and the power fluctuation of the tie lines. The exchange power and generation power of each sub-microgrid are coordinated and controlled, which are sent to the lower level model as scheduling instructions. The lower layer takes a single microgrid as the research object. By optimizing the output of power generation unit, the operation cost of single microgrid can be minimized. Iterative solution is adopted between the bi-level optimization models. In order to avoid the premature and local convergence of the algorithm in the optimization process, the improved artificial bee colony algorithm $(\mathrm{ABC})$ is used to solve the objective function. Finally, simulation examples verify the effectiveness and feasibility of the proposed strategy.
\end{abstract}

\section{Introduction}

Nowadays, the requirement for connecting the multiple microgrids, enhancing stability, improving energy management, optimization and promoting the ancillary services has received more and more attention. Microgrid cluster(MGC) consisting of multiple interconnected microgrids has great potential in improving microgrid reliability and reducing generating costs. The key to achieve global optimization and energy mutual-aid between microgrids is coordinated optimal scheduling strategy[1]. Recently, various approaches are proposed for coordinated operation and management of MGC distribution systems and different aspects of this concept are studied in the literature. In order to reduce the operation cost of the MGC, the regulation cost of each microgrid is modeled, so that each microgrid can assume the power regulation task according to its own situation [2]. A bi-level energy optimal scheduling model based on the microgrid economic scheduling is proposed focusing on the power coordinated scheduling among multiple microgrids[3]. Considering the advantages and application prospects of MGC, a bottom-up hierarchical control method is proposed, which can realize the economic operation of MGC[4]. The methods mentioned in the above literature, although improving the economic benefits of microgrid, their environmental benefits and power supply reliability are ignored.

Therefore, in order to improve the economic benefits and operational reliability of the MGC, this paper proposes a bi-level coordinated optimal dispatching strategy and uses an improved artificial bee colony (ABC) algorithm. Based on the original ABC algorithm, this algorithm introduces Gaussian mutation, strengthens the local search ability, and has higher search accuracy. The addition of chaotic disturbance overcomes the shortcomings of individuals being easily trapped in local optimization, and improves the robustness of the algorithm. Finally, simulation examples verify the effectiveness and feasibility of the proposed strategy.

\section{BI-LEVEL OPTIMIZATION MODEL FOR MICROGRID CLUSTER}

Multiple microgrids are connected by tie lines to form a MGC system. A typical structure of MGC system is shown in Figure. 1.The microgrid receives the dispatching instructions of the MGC, carries out power exchange through the tie line, and realizes the energy dispatching and mutual-aid. 


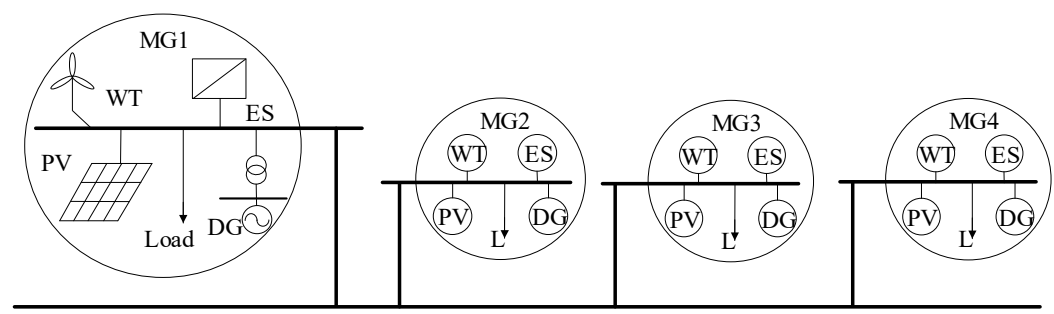

Figure. 1 A typical structure of microgrid cluster

\subsection{Upper Level Optimization Model}

The upper level optimization model of this paper is the MGC, and the objective function is established with the minimum operating cost of the MGC. The objective function of upper Level is as follows.

$$
\min f_{0}=\sum_{t=1}^{T}\left[C_{M G}(t)+C_{P L}(t)\right]=\sum_{t=1}^{T}\left[\sum_{i=1}^{M}\left[\alpha_{i} P_{M G, i}^{2}(t)+\beta_{i} \sum_{j=1}^{N} P_{M G, i}(t)+\gamma_{i}\right]+\sum_{i=1}^{M}\left[\lambda_{i} P_{T, i}^{2}(t)\right]\right]
$$

Where, the function $f_{0}$ is the total operating cost of the MGC; $C_{M G}(t)$ and $C_{P L}(t)$ are the cost of operating and power exchange between the microgrid; Tis the dispatch period and $\mathrm{t}$ is time interval. $P_{T L}(t)$ is the exchange power on the MGC tie line during the $t$ th time interval. $i$ is the $i$ th microgrid; . $\alpha_{i}, \beta_{i}$ and $\gamma_{i}$ are the output cost coefficients; $P_{M G, i}$ is the output power; $\lambda_{i}$ is the power exchange cost coefficient.

The constraints include system power balance constraint, microgrid output constraints, and tie line power constraints. The system balance constraint is shown as follows.

$$
\sum_{i=1}^{M}\left(P_{i}(t)+P_{T L, i}(t)\right)+P_{\text {load }}(t)+P_{\text {loss }}(t)=0
$$

Where, $P_{i}(t)$ is the total output power of all distributed power sources of microgrid $i$ during the $t$ th time interval. $P_{\text {load }}(t)$ and $P_{\text {loss }}(t)$ are the load and loss of the MGC during the $t$ th time interval.

The power output of the microgrid and tie line power constraint inequality are shown as follows.

$$
\begin{gathered}
P_{M G, i}^{\min } \leq P_{M G, i} \leq P_{M G, i}^{\max } \\
0 \leq P_{T L} \leq P_{T L}^{\max }
\end{gathered}
$$

Where, $P_{M G, i}^{\min }$ and $P_{M G, i}^{\max }$ are minimum and maximum power outputs of the $i$ th microgrid. $P_{T L}^{\max }$ is the maximum of the tie line power.

\subsection{Lower Level Optimization Model}

In this paper, the lower level optimization takes a single microgrid as the object. The objective function is established with the operating cost, the total power losses, the total emission and power fluctuation of the tie line.

Objective-1: Operating cost of a single microgri $\min f_{1}=\sum_{t=1}^{T}\left[C_{F}(t)+C_{o}(t)+C_{s}(t)\right]=\sum_{t=1}^{T}\left[C_{F}(t)+\sum_{j=1}^{N} K_{j} \cdot P_{j}(t)+\frac{1}{P_{J S h} 8760} \operatorname{Ccap} \cdot \frac{r(1+r)^{n}}{(1+r)^{n}-1} \cdot \sum_{t=1}^{24} P_{b a t}(t)\right]$
Where, $C_{F}(t), C_{o}(t)$ and $C_{S}(t)$ are the cost of fuel consumption operation and energy storage conversion.

The specific form of cost function is shown as follows.

$$
C_{F, F C}(t)=\frac{C_{n g}}{L_{H V n g}} \frac{P_{f c}(t)}{\eta_{f c}} ; \quad C_{F, D E}(t)=a P_{d e}^{2}(t)+b P_{d e}(t)+c
$$

Where, $C_{F, D E}$ is the generation cost, $a, b, c$ is the fuel cost coefficient. $C_{F, F C}$ is the generation cost of fuel cell. $C_{n g}, L_{H V n g}$ and $\eta_{f c}$ are the price of natural gas, low calorific value and efficiency. $K_{j}$ is the operation and maintenance cost coefficient. $P_{J S h}$, Ccap, $r$ are the rated power, the investment cost and depreciation rate. $n$ is the normal service life. $P_{b a t}(t)$ is the actual output.

Objective-2: Total power loss:

$$
\min f_{2}=\sum_{t=1}^{T} \sum_{i=1}^{N_{b r}}\left(R_{i} \cdot\left|I_{i}(t)\right|^{2} \cdot \Delta t\right)
$$

Where, $R_{i}$ and $I_{i}$ are the resistance and current of the $i$ th branch, and $N_{b r}$ is the number of branches.

Objective-3: Power fluctuations of the tie line:

$$
\min f_{3}=\sum_{t=1}^{T}\left|P_{T L}(t+1)-P_{T L}(t)\right|
$$

Where, $f_{3}$ is the power fluctuation of the tie line of the microgrid. $P_{T L}(t)$ is the exchange power

Objective-4: Pollutants emission: $\min f_{4}(X)=\sum_{t=1}^{T} E(t)=\sum_{t=1}^{T}\left(E_{F C}(t)+E_{D E}(t)+E_{W T}(t)+E_{P V}(t)\right)$

Where, $E_{F C}(t), E_{D E}(t), E_{W T}(t)$ and $E_{P V}(t)$ are the emissions of pollutants from fuel cell, diesel generator.

The constraints for lower level optimization objects include power balance constraints within a single microgrid, constraints on distributed power output and unit climbing rate.

The inequality constraints of the under level optimization model are shown as follows.

$$
P_{j}^{\min } \leq P_{j} \leq P_{j}^{\max } ;\left|P_{j, t}-P_{j, t-1}\right| \leq R_{j}
$$

Where, $P_{j}^{\min }, P_{j}^{\max }$ are minimum and maximum power outputs of the $j$ th distributed power in the microgrid. $R_{j}$ is the maximum climbing rate of the distributed power source. 


\section{IMPROVED ABC ALGORITHM AND ITS APPLICATION IN THE MODEL SOLUTION}

\subsection{Improved ABC Algorithm Based on Gaussian Mutation and Chaotic Disturbance}

The Gaussian mutation is described as follows. The original parameter value is replaced by a random number that matches a normal distribution with a mean of $\mu$ and a variance of $\sigma^{2}$. The variation formula is shown as follows.

$$
M(x)=x(1+N(0,1))
$$

where, $x$ is the original solution. $N(0,1)$ is a random number that conforms to a normal distribution. $M(x)$ is the value after Gaussian mutation.

Using Gaussian mutation can improve the local search ability of the algorithm. It can enhance the robustness of the algorithm while finding the global minimum point quickly and accurately.

Chaotic disturbance can use the ergodicity and randomness of chaotic variables to generate chaotic sequences randomly based on the solution. In order to make the algorithm jump out of the limit and continue to search, the chaotics sequence is used to disturb the individuals that cause the search stagnation.

Considering that the value of the Tent map is relatively gentle, the Tent map is selected to generate chaotic sequences. Tent mapping formula is shown as follows.

$$
X_{d}=\left\{\begin{array}{ll}
2 x_{d}, & 0 \leq x_{d} \leq 1 / 2 \\
2\left(1-x_{d}\right), & 1 / 2 \leq x_{d} \leq 1
\end{array} d=1,2, \cdots, D\right.
$$

where, $X_{d}$ is the d-dimensional vector of the chaotic sequence $X . x_{d}$ is a random number that obeys a uniform distribution, $x_{d} \in[0,1]$.The steps of chaotic disturbance are described as follows.

Step1: applying Tent mapping produces chaotic variables.

Step2: According to equation (13), the chaotic variable is mapped to the solution space.

$$
\text { new } X_{d}=\min _{d}+\left(\max _{d}-\min _{d}\right) X_{d}
$$

where, $\max _{d}$ and $\min _{d}$ are the maximum and minimum values of the d-dimensional vector, respectively.

Step3: Chaotic disturbance of individuals is carried out according to equation (14).

$$
\operatorname{new} X^{\prime}=\left(X^{\prime}+\operatorname{new} X\right) / 2
$$

Where, $X^{\prime}$ is the individual trapped in the local optimum. new $X$ is the chaotic disturbance momentum generated randomly. new $X^{\prime}$ is the individual after disturbance.

\subsection{Application of Improved ABC algorithm for Multi-objective optimization problem}

The specific steps of using the improved $\mathrm{ABC}$ algorithm to solve the multi-objective optimization problem are as follows.

Step 1: Initialize, input the basic parameters of the MGC. Set the population size of bee is 50, and the number of iterations is $100, c_{1}=c_{2}=2.1$.

Step 2: According to equation (15), the $\mathrm{N}$ individuals are generated.

$$
x_{i, j}=x_{j}^{\min }+\operatorname{rand}(0,1) \cdot\left(x_{j}^{\max }-x_{j}^{\min }\right)
$$

Where, $i=1,2, \ldots, N$ is a D-dimension vector. $j=1,2, \ldots, d$.

Step 3: Calculate the fitness value for food souse by using equation(16). It is updated local optimal position and global optimal position by using equation(17).

$$
\begin{gathered}
\text { fit }_{i}=\left\{\begin{array}{l}
\frac{1}{1+f_{i}}, f_{i}>0 \\
1+\left|f_{i}\right|, f_{i} \leq 0
\end{array}\right. \\
p_{i}=\frac{f_{i} t_{i}}{\sum_{j=1}^{N} f i t_{j}}
\end{gathered}
$$

Where, $f_{i}$ is the function value of the $i$ th solution.

Step 4: Build the repository. All Pareto solutions are stored in the repository.

Step 5: Find a new solution by using equation (18) The optimal solution is selected by comparison, and the corresponding individual position is determined.

$$
\text { new } X_{i}^{j}=X_{i}^{j}+\operatorname{rand}(-1,1)\left(X_{i}^{j}-X_{k}^{j}\right)
$$

Step 6: If the number of times to find a new solution reaches the maximum, and no better solution is found, a random solution is generated to replace the previous solution.

Step 7: Calculate the fitness value and average fitness value of the individuals in the colony. If the individual fitness is greater than the average fitness, calculate according to equation (13); otherwise calculate according to equation (14). If the performance after mutation and perturbation is better, replace the previous individual; otherwise keep the original individual unchanged.

Step 8: Updating the repository. In this step the repository is updated so that all solutions in the repository would be Pareto optimal solutions.

Step 9: Output the final result after the maximum number of iterations; otherwise return to step4 and continue execution.

\section{SIMULATION RESULTS}

Four microgrids constitute the MGC structure, as shown in Figure 1. A single microgrid consists of WP, PV, DE, ES and loads. The specific parameters of the microgrids and their related power generation units are shown in Table 1and Table 2. 
Table 1. Unit configuration and output cost coefficient of Microgrid

\begin{tabular}{ccccccccc}
\hline MG & WT(kW) & PV(kW) & $\mathrm{DE}(\mathrm{kW})$ & $\mathrm{FC}(\mathrm{kW})$ & $\mathrm{ES}(\mathrm{kW})$ & $\alpha_{i} /\left(\right.$ yuan $\left./ \mathrm{kW}^{2}\right)$ & $\beta_{i} /($ yuan $/ \mathrm{kW})$ & $\gamma_{i} /$ yuan \\
MG1 & 100 & 60 & 300 & 250 & 350 & 0.00595 & 0.140 & 0 \\
MG2 & - & 80 & 300 & 250 & 350 & 0.001196 & 0.175 & 0 \\
MG3 & 80 & 60 & 300 & 250 & 350 & 0.001165 & 0.175 & 0 \\
MG4 & 100 & - & 250 & 250 & 350 & 0.000595 & 0.155 & 0 \\
\hline
\end{tabular}

Table 2. Microgrid output and DE cost coefficient

\begin{tabular}{ccccccc}
\hline $\mathrm{DE}$ & $a_{\mathrm{i}} /\left(\right.$ yuan $\left./ \mathrm{kW}^{2}\right)$ & $\mathrm{b}_{\mathrm{i}} /($ yuan $/ \mathrm{kW})$ & $\mathrm{c}_{\mathrm{i}} /$ yuan & $C_{n g}\left(\right.$ yuan $\left./ \mathrm{m}^{3}\right)$ & $L_{H V n g}\left(\mathrm{kWh} / \mathrm{m}^{3}\right)$ & $\eta_{f c}$ \\
1 & 0.0168 & 0.168 & 0.3 & 2.5 & 7.9 & $60 \%$ \\
\hline
\end{tabular}

The iterative convergence curve of MGC operating costs is shown in Figure. 2. It can be seen from the operating results that the original $\mathrm{ABC}$ algorithm needs 80 iterations to converge to the minimum value, which is 15424.86 (yuan). The chaotic artificial bee colony(CABC) algorithm iterates about 68 times to converge to the minimum value of 15379.62 (yuan). The improved

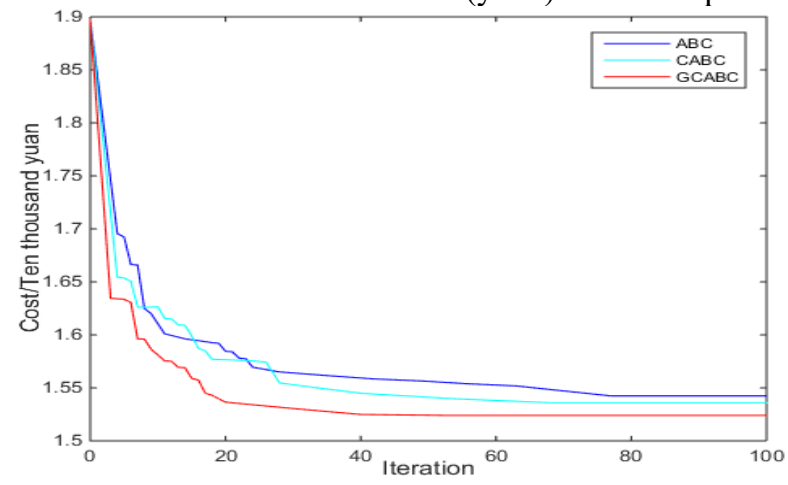

Figure. 2 Iterative convergence curve of MGC operating cost

In Table 3, comparing the optimization results of the $\mathrm{ABC}, \mathrm{CABC}$ and $\mathrm{GCABC}$ algorithm, the non-dominated artificial bee colony optimization algorithm based on Gaussian mutation and chaotic disturbance (GCABC) can converge to a minimum value of 15246.19 (yuan) after about 50 iterations. It is can be seen obviously that the proposed GCABC optimization algorithm has faster convergence speed, smaller convergence value, and better effect.

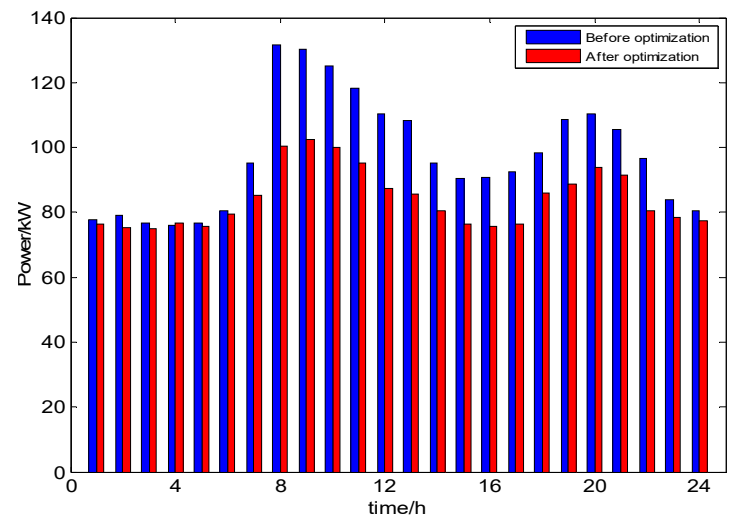

Figure. 3 Power fluctuation of tie line

solutions that represent the best solutions of objective functions are given.

Table 3. Comparison of the results of the three optimization methods

\begin{tabular}{ccccc}
\hline $\begin{array}{c}\text { Objective } \\
\text { function }\end{array}$ & Method & Average & $\begin{array}{c}\text { Worst } \\
\text { solution }\end{array}$ & $\begin{array}{c}\text { Best } \\
\text { solution }\end{array}$ \\
Cost(yuan) & GCABC & 4358.335 & 4359.06 & 4357.61 \\
& CABC & 4460.045 & 4486.52 & 4433.57 \\
& ABC & 5319.515 & 5973.25 & 4665.78 \\
$P_{\text {Loss }}(k W h)$ & GCABC & 91.335 & 91.46 & 91.21 \\
& CABC & 101.155 & 103.75 & 98.56 \\
& ABC & 124.435 & 136.49 & 112.38 \\
Power & GCABC & 502.29 & 502.76 & 501.82 \\
fluctuation $(k W)$ & CABC & 573.78 & 597.83 & 549.73 \\
& ABC & 672.865 & 732.47 & 613.26 \\
Emission $(k g)$ & GCABC & 6144.01 & 6145.19 & 6142.83 \\
& CABC & 6496.615 & 6673.28 & 6319.95 \\
& ABC & 7697.646 & 8356.72 & 7038.57 \\
\hline
\end{tabular}

To show the improvement of each objective function with respect to the other objective functions, the three-dimensional (3D) plot of the Pareto-optimal solutions of the multi-objective problem are shown in Figure4. In this problem with four objective functions, four three-dimensional Pareto front would be obtained with the objectives emission, cost, power loss and Power fluctuation. The best solutions for objectives emission, cost, power loss and power fluctuation are $6142.8(\mathrm{~kg})$, 4357.6 (yuan), 91.21 (kWh) and $501.8 \quad(\mathrm{~kW})$, respectively. 

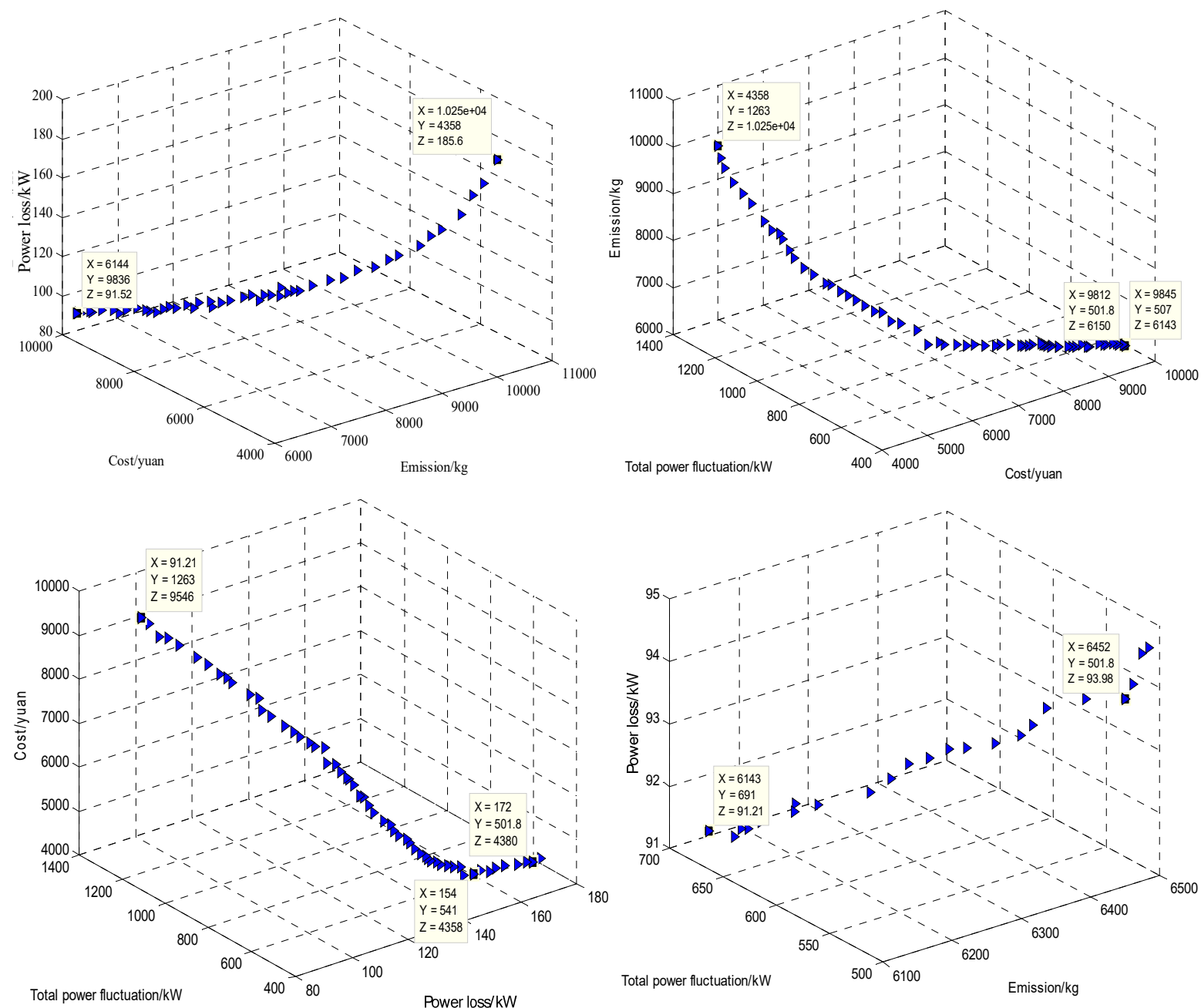

Fig. 4 Three-dimensional(3D) plot of the Pareto-optimal solutions of the objective function

\section{CONCLUSION}

This paper proposes a bi-level coordinated optimization strategy for a MGC system. Through the coordinated control of the output of each microgrid, the operating cost of the entire system is reduced. Considering the economic benefits and operation reliability of a single microgrid, the mathematical models of microgrid operation cost, tie line power fluctuation, network loss and pollutant emission are established. Aiming at the established mathematical model, an improved artificial bee colony optimization algorithm based Gaussian mutation and chaotic disturbance is used to solve the problem. Compared with the original $\mathrm{ABC}$, the chaotic artificial bee colony(CABC) algorithms, the numerical simulation results shown that the proposed algorithm has faster convergence speed and smaller convergence value, which can prevent the algorithm from falling into local optimization and particle premature problems. The strategy proposed in this paper has effectively reduced the operating costs of MGC and improved the economic benefits of the grid.

\section{ACKNOWLEDGMENTS}

This work was supported by the National Natural Science Foundation of China (No.62063016), the Science and Technology Plan of Gansu Province (No.20JR10RA177).

\section{REFERENCES}

1. Farzin H, Fotuhi-Firuzabad M, Moeini-Aghtaie M. Role of Outage Management Strategy in Reliability Performance of Multi-Microgrid Distribution Systems, IEEE Transactions on Power Systems, 2018, 33(3): 2359-2369.

2. Han Y, Zhang K, Li H, et al. MAS-based distributed coordinated control and optimization in microgrid and microgrid clusters:a comprehensive overview[J]. IEEE Transactions on Power Electronics, 2018,33(8): 6488-6508.

3. Cheng Yifan, Qiao Fei, Hou Ke, et al Research on bi-level energy dispatching strategy optimization for regional microgrid cluster[J], Chinese Journal of Scientific Instrument, 2019, 40(5): 68-77.

4. Tian P, Xiao X, Wang K, et al. A Hierarchical 
Energy Management System Based on Hierarchical Optimization for Microgrid Community Economic
Operation[J]. IEEE Transactions on Smart Grid, 2015, 7(5):2230-224. 